FERMILAB-FN-1095-ND

\title{
2 A Study of Dielectric Breakdown through Argon in a . Uniform Electric Field
}

\section{S. Lockwitz; H. Jostlein}

ABSTRACT: This paper details a study of the dielectric breakdown of liquid argon between two

5 plates in a nearly uniform field. In this geometry, effects of stressed volume independent of area can be probed. Additionally, liquid argon purity effects are considered.

6 KeYwords: Liquid Argon; Time Projection Chambers; Dielectric Strength, Electric Breakdown;

7 High Voltage.

\footnotetext{
${ }^{*}$ Corresponding author: lockwitz@fnal.gov (S. Lockwitz)
} 


\section{Contents}

10 1. Motivation 囵

11 2. Experiment Setup \& Procedure 2]

12 3. Results 3

13 B.1 Breakdown vs. Distance

14 B.1.1 Volume vs. Area

15 B.2 Breakdown vs. Contamination Level

16 3.2.1 Breakdown vs. Pressure

$\begin{array}{llll}17 & 3.3 & \text { Breakdown vs. Number of Breakdowns }\end{array}$

18 田. Gaseous Argon 8

19 5. Discussion \& Conclusion 9

20 6. Acknowledgements

\section{Motivation}

Liquid Argon (LAr) time projection chambers (TPCs) are becoming a preferred tool to study neutrino interactions. TPCs require an electric field to move ionization electrons to a detection plane. For large detector mass, one employs substantial drift length, and therefore needs high voltage to provide the required drift field. It has not been uncommon for such detectors to encounter difficulty maintaining these voltages without electrical instabilities. The electrical breakdown properties of LAr have been measured over small distance scales for a long time [1]; only recently have these studies been carried out over the distance scale of interest [2, 3, 价. Typically the breakdowns occur at lower electric fields the greater the electrode gap and the larger the device.

Early attempts to understand and predict spark voltages between gaps [5] have proposed a model based on random statistical initiations, which were used to predict breakdown as a function of stressed size.

A recent study [四] measured the sustainable field strength in ultra-pure LAr for a variety of electrode sizes and spacings. The results were presented as a function of "stressed area," i.e. the area on the cathode surface that is subjected to $90 \%$ or more of the peak electric field. The cathodes were spheres of a range of sizes separated by variable gaps from an anode plane. Due to the strong correlation of area with volume for this sphere geometry, it was not considered possible to extract the relative role of stressed area and volume. 
We have constructed a new cryostat with an electrode system that allows a change of the gap volume while keeping the stressed area nearly constant. This was achieved by using a Rogowskiinspired set of nearly flat electrodes. The LAr was filtered at the Fermilab PAB facility to a purity in the ppb range, with electron life time in the few 10s of $\mu$ s range.

Subsequently, the LAr was intentionally doped with increasing levels of oxygen to understand the sensitivity of the breakdown field to contamination.

\section{Experiment Setup \& Procedure}

The cryostat was first filled with pure liquid argon. The argon started off as scientific grade with a few ppm of oxygen. It was first passed through a molecular sieve to remove water and then activated-copper-coated granules to remove oxygen. Details of the filteration system can be found in Reference [6]. The resulting liquid was roughly 8 ppb oxygen argon as measured by a Servomex DF-560e gas analyzer.

To explore the effect of contaminants on high voltage performance, a custom injection system was used. A known concentration of argon plus contaminant gas was added to a 3.74 liter bottle to a target pressure. The bottle was then bled off into the main cryostat to a pressure to reach the desired impurity level. Over the duration of the experiment, residual gas analyzers were available to monitor the impurity levels.

The dielectric strength tests were performed in Blanche: the Breakdown in Liquid ArgoN Cryostat for High voltage Experiments. Blanche is a $150 \mathrm{~cm}$ tall, $76 \mathrm{~cm}$ inner diameter vacuum insulated cryostat. A condenser is used to condense the gaseous argon boil off and return it to the main volume through a molecular sieve to remove the water condensed in the gas phase. This allowed for long-term running.

The high voltage system of the setup consists of a power supply, high voltage cables, a lowpass filter, and a feedthrough. The power supply was a Glassman LX150N12 supply capable of $-150 \mathrm{kV}$ [7]. A Glassman-supplied cable (DS-2121) brought the high voltage into a electric filter. The filter consists of a $75 \mathrm{M} \Omega$ resistor network submerged in transformer oil. The purpose of the filter is two-fold: along with the capacitance in the downstream cable, it reduces the voltage ripple from the power supply, and it partitions the stored energy in the system.

From the filter, a Dielectric Sciences 2134 cable delivers the high voltage to the feedthrough. Near the end of the cable, the ground braid has been removed and a custom housing holding a Pearson 110 current transformer has been installed to detect electrical discharges. The ground of the system is maintained on the outside of the housing.

The feedthrough is a custom device and it is described elsewhere [8]. Briefly, it consists of an outer ground tube encasing a $5 \mathrm{~cm}$ tube of UHMW PE with a $2.5 \mathrm{~cm}$ center conductor in the center. The feedthrough has been pressure tested and is leak tight. It is only rated to $-100 \mathrm{kV}$ setting the upper limit for the testing presented here.

At the base of the feedthrough, a Rogowski-inspired plate made of stainless steel was attached. An image of it is shown in Figure 11. This plate served as the cathode in our testing. The ground plate was a $71 \mathrm{~cm}$ diameter stainless steel plate held in place by three tubes attached to the top plate of the cryostat. One of the tubes was instrumented with an force sensor. The feedthrough and cathode plate were attached to the cryostat top plate through a vertical translator equipped with 


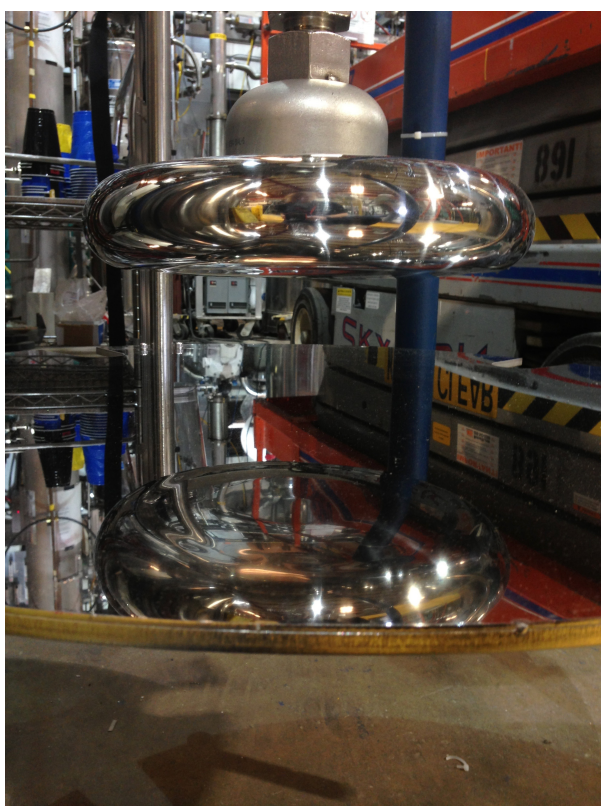

(a) lyzed with a RoOT [0] software program.

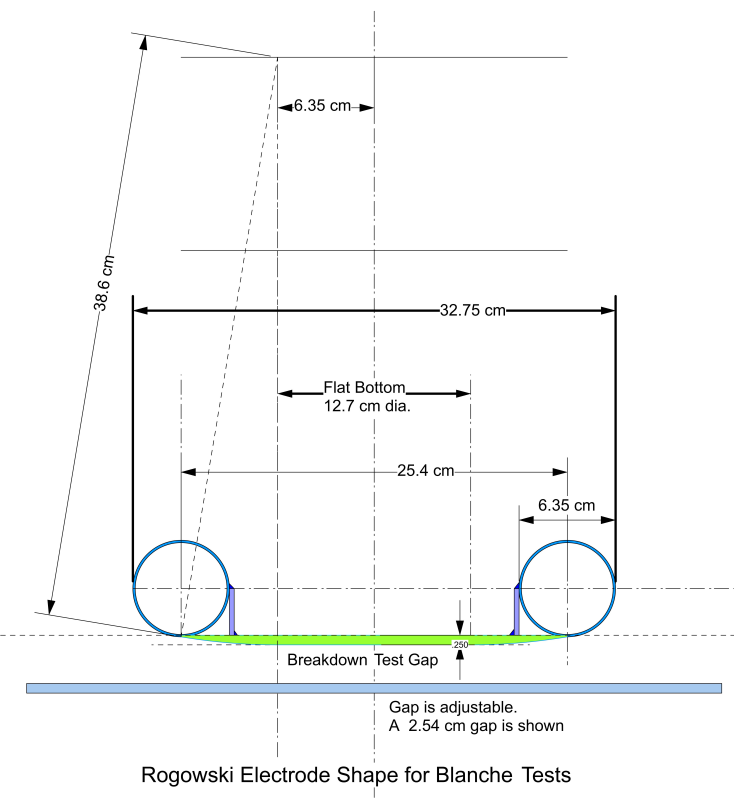

(b)

Figure 1. Left: Photo of the Rogowski-inspired plate. The reflection of the plate can be seen in the ground plane. Right: Drawing of the plate.

edge-welded bellows. The cathode plate could be raised and lowered by the motorized translator.

Once a desired gap spacing between the cathode and ground plates was set, a LABVIEW program paired with an NI-6216 box raised the voltage of the power supply, stopped after receiving a discharge signal, recorded the information to a log file, and waited a prescribed amount of time before ramping again. The Glassman was controlled by analog outputs from the NI-6216. The breakdown signal came from the toroid, but was amplified and stretched to allow for detection within the setup's $32 \mathrm{kHz}$ across seven channels sampling rate. The resulting log files were ana-

plane. Right: Drawing of the plate.

For each gap distance, between 130 and 2091 breakdowns were recorded. The breakdown values including current and toroid signals were analyzed to remove any false triggers. The voltages for a given distance were then plotted in time to check for any drift. If stable, the data were then histogrammed using a RоOT program and fit with a Weibull function [5, [10]. Means and widths were taken from this fit.

\section{Results}

\subsection{Breakdown vs. Distance}

For the purest argon evaluated in this test, the breakdown voltages versus gap distance are shown in Figure [ (a). This argon was measured to have less than $10 \mathrm{ppb}$ oxygen as measured with a gas analyzer capable of measuring oxygen concentrations down to $<0.1 \mathrm{ppb}$. The water concentration 


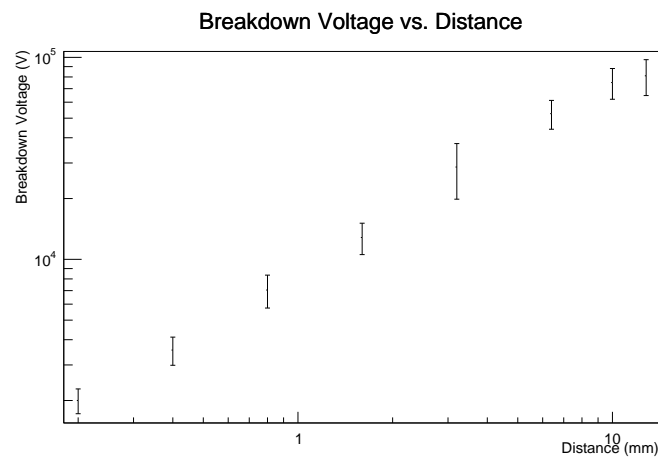

(a) maximum field as calculated by the FEA.

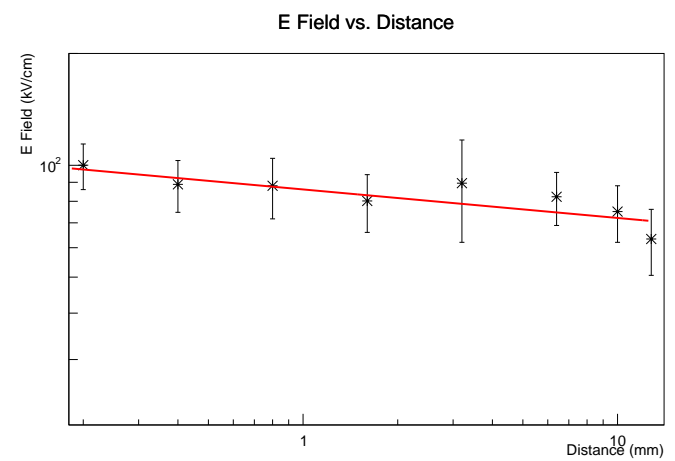

(b)

Figure 2. Breakdown voltages and resulting electric fields for pure liquid argon versus distance.

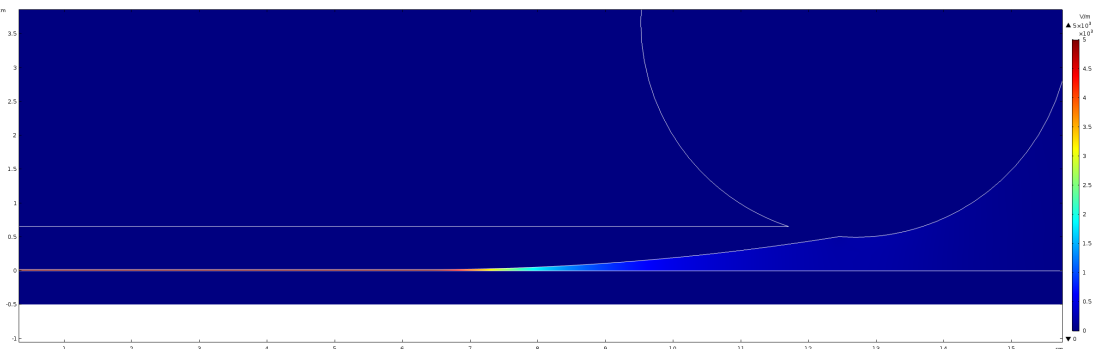

(a)

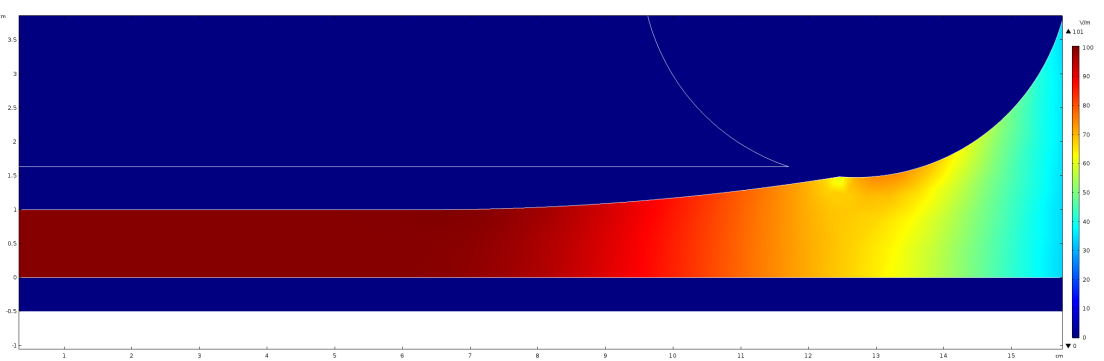

(b)

Figure 3. The electric field as calculated by FEA for the 0.2 and $10 \mathrm{~mm}$ gaps.

was not measured. The resulting approximately uniform electric field versus distance is shown in Fure 2(b). Field uniformity in the gap region was a goal of the test, however fabrication issues led to a slight recession around the plate. An Finite Element Analysis (FEA) of the setup shown in Figure 3 shows the field is generally uniform in the volume within the $6.35 \mathrm{~cm}$ radius disc and has gap-dependent variations that increase radially. In Figure 2 (b), the reported electric field is the 
links. The literature, however, states that the parameter of interest could be either area or volume. Previous results in liquid argon [ [ [12] have related the maximum field at breakdown to an area with an electric field at least $\varepsilon$ percent of the maximum field. This value is called the stressed area, and $\varepsilon$ values are typically 80 or $90 \%$. The aforementioned studies showed a power law dependence of $E_{\max }=C(A)^{-0.26}$ where $C$ is a constant, and $A$ is the stressed area. These studies, however, used a sphere-plate geometry where the stressed area was strongly correlated with the stressed volume making it difficult to distinguish the relevant parameter.

Here, the plate geometry allows for an increase in stressed volume as the gap distance is increased while keeping the stressed area roughly constant. For instance, when changing the gap from 0.2 to $10 \mathrm{~mm}$, the stressed area at the $90 \%$ (80\%) level increases a factor of 1.9 (2.4), while the stressed volume at the $90 \%(80 \%)$ level increases a factor 93 (121). Illustrating this feature is Figure $\emptyset$ where the stressed areas and volumes are shown for different gap distances explored in this study. Examples of calculated electric fields for the 0.2 and $10 \mathrm{~mm}$ gaps spacings are shown in Figure 3 .

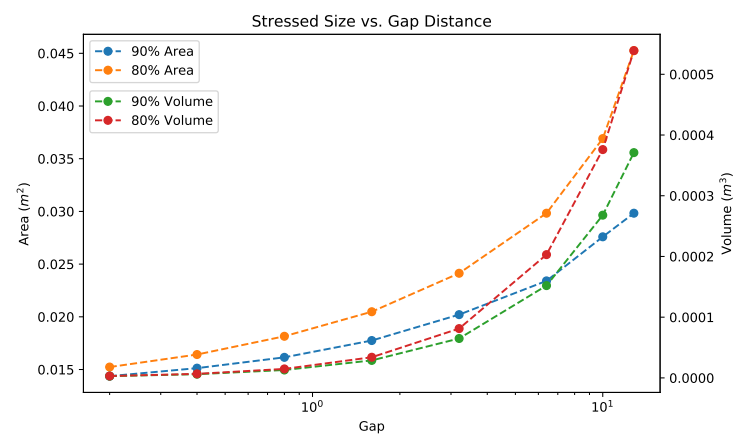

Figure 4. Stressed areas and volumes with $\varepsilon$ equal to 80 and $90 \%$ versus distance as calculated from an FEA.

Figure 5 shows the maximum electric field versus stressed area and volume from this study along with data from an earlier ball-plate geometry [四]. Only data with $\mathrm{O}_{2}$-equivalent purities less than $10 \mathrm{ppb}$ were considered from the earlier study. The data points represented by black triangles are from measurements done with a $1.3 \mathrm{~mm}$ diameter sphere above a grounded plane at various distances. The cluster of points at high $E_{\max }$ and high stressed area or volume are from when the ball was many radii away from the ground plane such that the entire surface area was stressed. Since the stressed area was not free to increase, the test at that point was no longer evaluating the stressed parameters under study. The data were fit to evaluate which parameter best described behavior across geometries, but the cluster of aforementioned $1.3 \mathrm{~mm}$ data was excluded. The function was $E=C d^{m}$ where $d$ is the stressed size of interest. The results are summarized in Table 1 along with fits to the ball-only points in parentheses. The fit of the maximum electric field versus stressed volume at the $90 \%$ level best unified the data however, the fit deviates from the points at larger volumes suggesting another effect noticeably contributes for large systems, or there is some feature .

The literature [11] has suggested a minimum electric field needed for breakdown. In the earlier sphere-plate data, this value was $38 \mathrm{kV} / \mathrm{cm}$. Here, a breakdown was observed with a maximum 


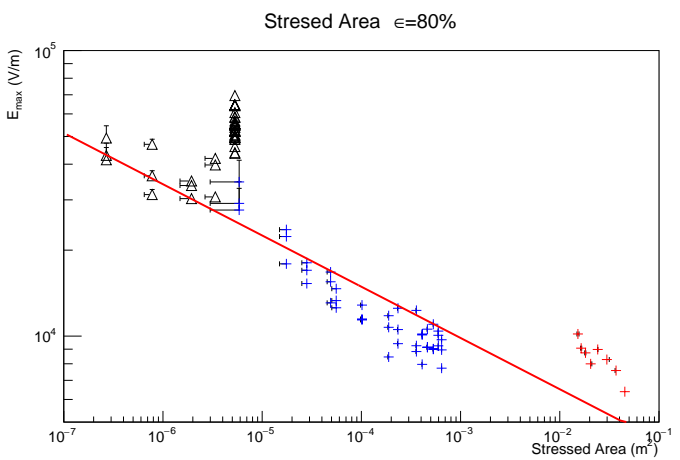

(a)

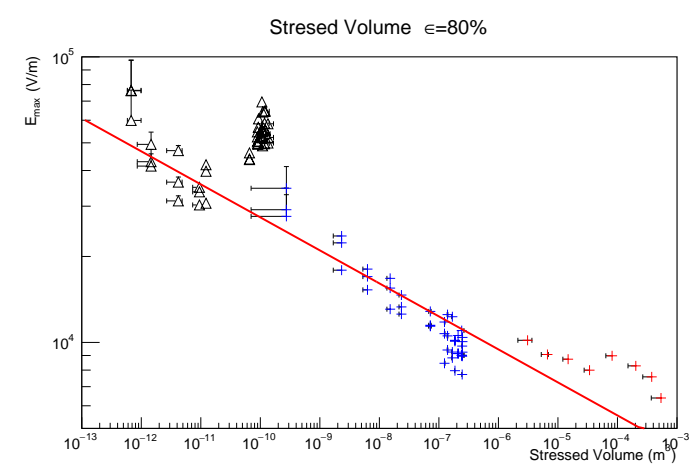

(c)

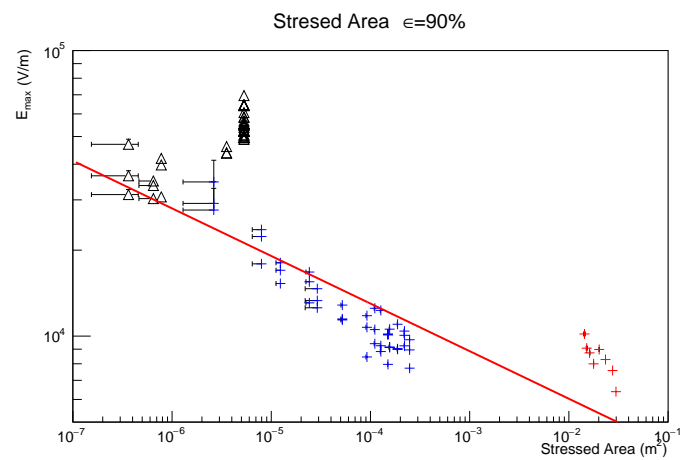

(b)

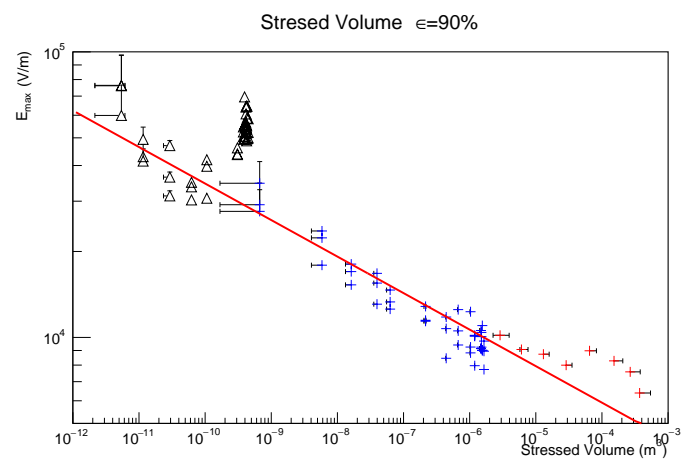

(d)

Figure 5. Breakdown electric field versus stressed geometry for the plate data and earlier sphere data. The black triangle points are from data with a $1.3 \mathrm{~mm}$ diameter sphere, the blue crosses are from a $76 \mathrm{~mm}$ diameter sphere, and the red crosses are from this study. The black triangle points clustered around a high $E_{\max }$ and higher stressed area or volume are excluded from the fit.

\begin{tabular}{|l|r|r|r|}
\hline$E_{\max }$ vs. Parameter $(\varepsilon)$ & Fit Intercept & Fit Slope & $\chi^{2} /$ dof \\
\hline Area $(80 \%)$ & $3.46(3.21)$ & $-0.180(-0.229)$ & $0.011\left(4.1 \times 10^{-3}\right)$ \\
Area $(90 \%)$ & $3.45(3.14)$ & $-0.167(-0.224)$ & $0.014\left(4.3 \times 10^{-3}\right)$ \\
Volume $(80 \%)$ & $3.28(3.08)$ & $-0.116(-0.137)$ & $0.009\left(4.3 \times 10^{-3}\right)$ \\
Volume (90\%) & $3.26(3.14)$ & $-0.128(-0.141)$ & $0.006\left(3.7 \times 10^{-3}\right)$ \\
\hline
\end{tabular}

Table 1. Results from fitting the log-log data. Values in parentheses are from fits excluding the plate data.

electric field as low as $22.9 \mathrm{kV} / \mathrm{cm}$. This reduction in minimum field suggests that size effects are still dominating an attempt at evaluating the minimum electric field needed for breakdown in these studies. Electric field calculations in both of these studies assume ideal geometries ignoring any microscopic imperfections on surfaces or particles in the liquid. The studies are also insensitive to time effects.

\subsection{Breakdown vs. Contamination Level}

The literature [13] also mentions an effect of oxygen contamination level in the liquid argon on 
breakdown voltage. Oxygen, water, and nitrogen are the primary contaminants of interest in neutrino LArTPC experiments. Oxygen and water are electronegative and reduce the signal electrons. Nitrogen diminishes the secondary scintillation light. Here, oxygen was added in varying quantities up to $800 \mathrm{ppb}$ and measured with a gas analyzer. At the last oxygen contamination level, $5 \mathrm{ppm}$ of nitrogen were added and also measured with an analyzer. Water, with its significantly higher freezing and boiling temperatures, was not considered due to the technical challenge of introducing it to the bulk liquid and successfully measuring it.

The breakdown maximum electric fields versus distance are shown in Figure 6(a). In the lower plot of the figure, a ratio to the breakdown value at that distance in pure argon is given. In Figure 6(b), the average of this ratio for a given contamination level is reported as a function of added impurity level. The linear fit does not include the nitrogen contamination point shown with blue dashed lines.

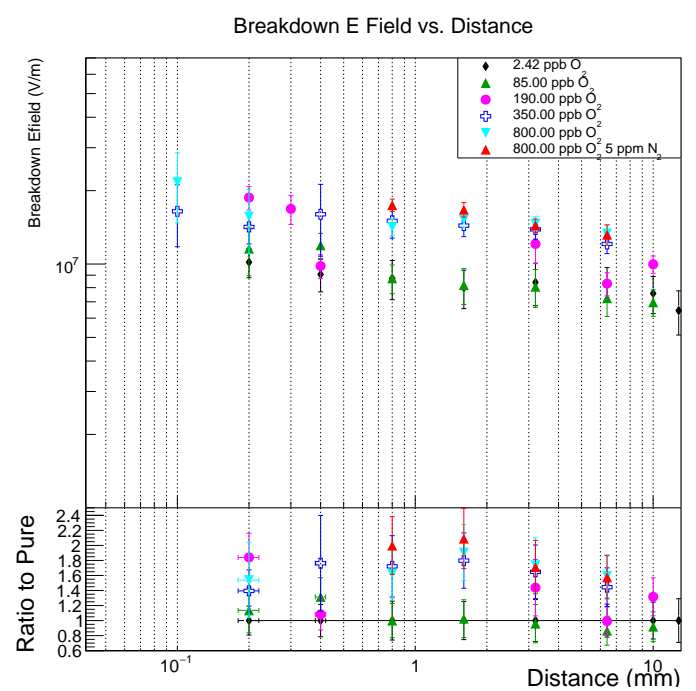

(a)

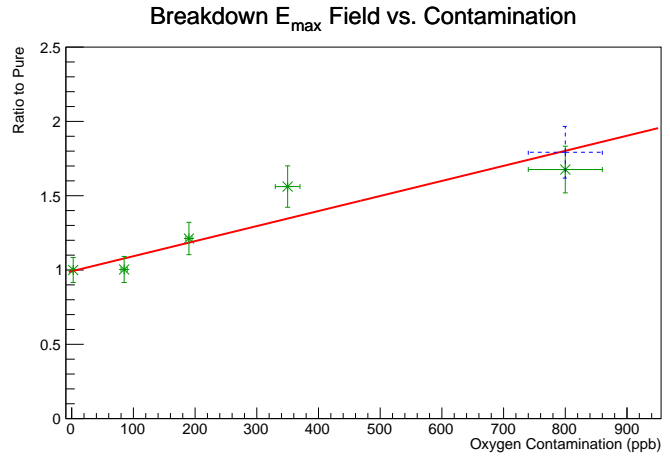

(b)

Figure 6. This should probably be E field.

\subsubsection{Breakdown vs. Pressure}

The data presented in this section were collected with a 8 psig pressure reading in the gas space of the cryostat. The level remained largely constant during running. A special set of runs was recorded with the gas space at $18 \mathrm{psig}$, however no significant change in breakdown behavior was observed.

\subsection{Breakdown vs. Number of Breakdowns}

Over the course of running, in some cases, it was noted that the breakdown voltage had a dependence on the number of breakdowns. The noted effect was that the breakdown voltage for a given spacing would start off low and increase until plateauing to a higher value as shown in Figure 7 . The effect was not a regular feature of the data. It seemed to be dependent on the amount of time between runs (i.e. time without a breakdown), and was more apparent when increasing gap spacing. 


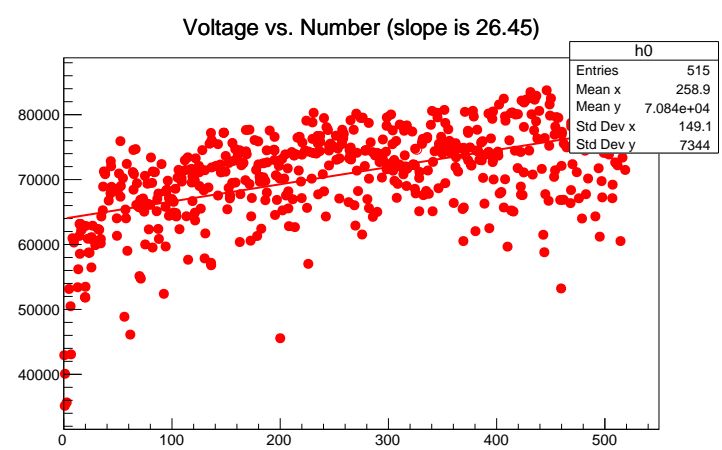

(a) presented in Section 3 .

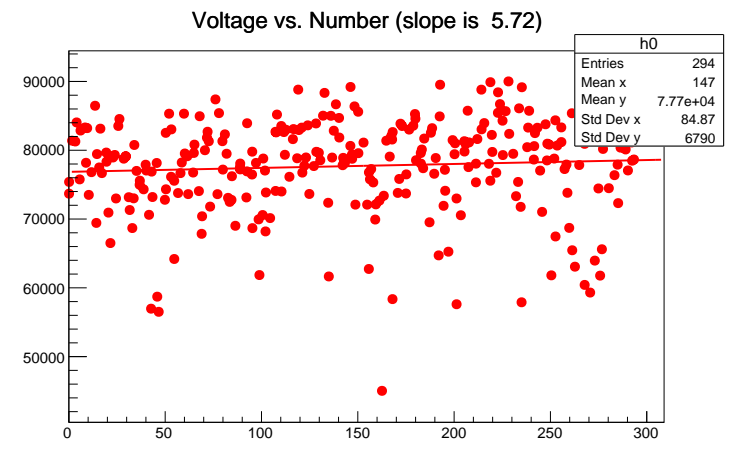

(b)

Figure 7. Above is the breakdown voltage versus breakdown number for a $6.4 \mathrm{~mm}$ gap in about $350 \mathrm{ppb}$ liquid argon. On the left, the run has a turn-on feature on the beginning; on the right, the voltage is comparatively stable in time.

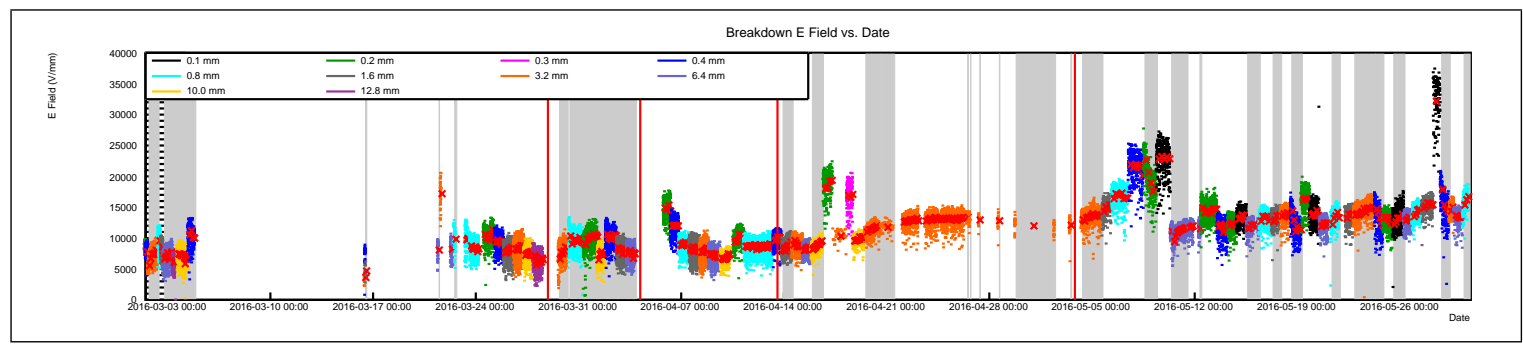

Figure 8. The breakdown voltage divided by the gap distance over the runs. The vertical red lines are points were contamination was added to the system. The data with a gray box behind it was not used in the analyses in the other sections of the paper.

The intensity of previous discharges was investigated as a dependent variable, but it did not uniormily affect later points. It is worth noting that only stable runs were considered in the analysis 


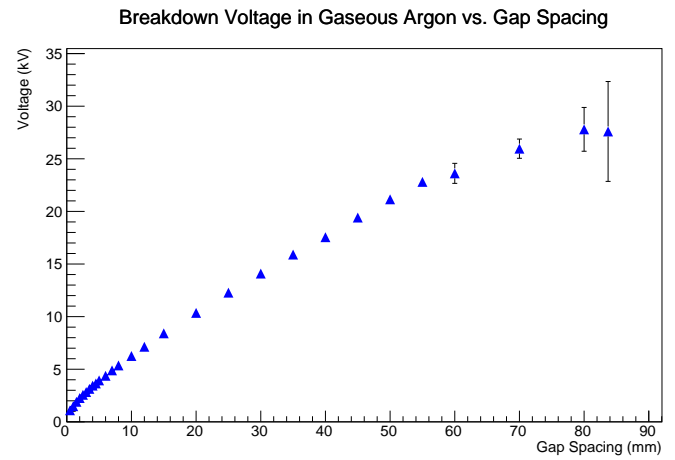

(a)

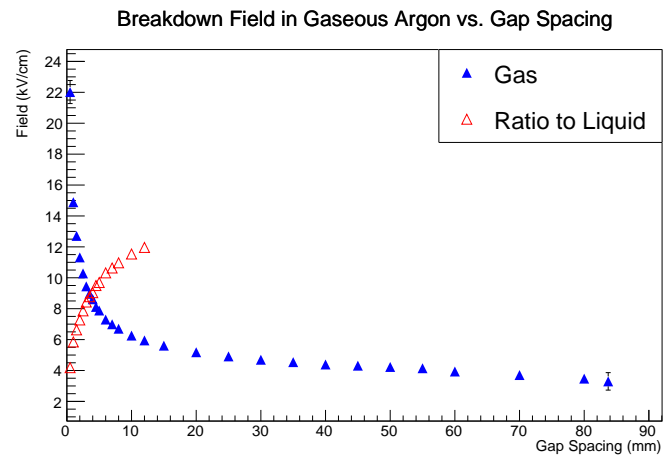

(b)

Figure 9. Breakdown measurements in commercial argon gas.

\section{Discussion \& Conclusion}

In this paper, it was shown that the size dependence on peak electric field at breakdown is more correlated with stressed volume than area. If one assumes breakdown is initiated at a surface, this suggests that there needs to exist a field to develop the instability into a discharge. While a relationship between breakdown field and size is presented, it is unlikely that the trend can be extended to very large sizes.

The authors note that this study does not consider all electrical instabilities, but rather only sudden discharges. Other phenomena, such as extended current draws in time not resulting in a discharge, have now been observed in other setups.

As earlier literature suggested and demonstrated, oxygen contamination does effect the electrical performance in liquid argon.

An effect was noted in a later test setup while this manuscript was being prepared. The setup was also in the Blanche cryostat, however, a purity monitor based on [14] was also installed. After a sudden high voltage discharge in the setup, a drastic drop in purity was noted. This can be seen in Figure 10 where a slow degradation in purity was being studied when there was a sudden drop after a discharge event. The purity monitor cannot identify what the contaminant was and the sudden drop did not correspond to a change on the oxygen analyzers.

One thought was that the effect could be due to positive ions created during the discharge. This idea was rejected after it was found that the recovery time did not change when the electric field was turned off. The current favored hypothesis is that the discharge liberates water into the bulk liquid from metallic surfaces in the cryostat and over time, the water eventually plates out again.

The authors believe this feature affected the data causing the irregularities described in Section 3.3, and possibly the comparitively higher breakdown fields in Section 3.1.1. 


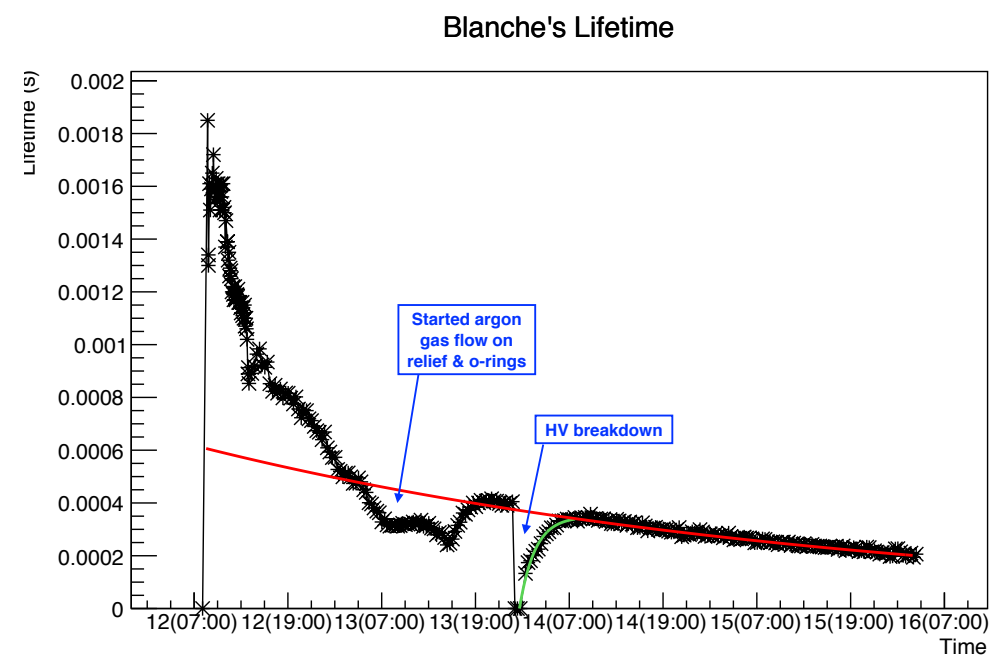

Figure 10. The decay of the liquid argon lifetime during an unrelated test. After a discharge, a drop in the purity as measured by a purity monitor was observed. The purity gradually recovered to its pre-breakdown state.

\section{Acknowledgements}

The authors wish to thank Simone Rizzardini for his cheerful help in collecting data, Stephen Pordes and Brian Rebel for their support, editorial contributions, and scientific discussions, and Jim Walton and Alan Hahn for their technical assistance.

Fermilab is operated by Fermi Research Alliance, LLC under Contract No. De-AC02-07CH11359 with the United States Department of Energy. 


\section{References}

[1] D. Swan and T. Lewis, "Influence of electrode surface conditions on the electrical strength of liquified gases,” J. Electrochem. Soc. 107 (1960) 180.

[2] A. Blatter et al., "Experimental study of electric breakdowns in liquid argon at centimeter scale," JINST 9 (2014) P04006, arXiv:1401.6693 [physics.ins-det].

[3] F. Bay et al., "Evidence of electrical breakdown induced by bubbles in liquid argon," arXiv:1401.2777 [physics.ins-det].

[4] R. Acciarri et al., "Liquid Argon Dielectric Breakdown Studies with the MicroBooNE Purification System," JINST 9 no. 11, (2014) P11001, arXiv:1408.0264 [physics.ins-det].

[5] W. Weibull, A Statistical Theory of the Strength of Materials. Ingeniörsvetenskapsakademiens handlingar. Generalstabens litografiska anstalts förlag, 1939.

[6] A. Curioni, B. Fleming, W. Jaskierny, C. Kendziora, J. Krider, S. Pordes, M. Soderberg, J. Spitz, T. Tope, and T. Wongjirad, "A regenerable filter for liquid argon purification," Nuclear Instruments and Methods in Physics Research Section A: Accelerators, Spectrometers, Detectors and Associated Equipment 605 no. 3, (2009) 306-311. http://www.sciencedirect.com/science/article/pii/s016890020900775X.

[7] Glassman High Voltage Inc., PO Box 317, 124 West Main Street, High Bridge, NJ 08829-0317, U.S.A.

[8] S. Lockwitz and H. Jostlein, "A study of dielectric breakdown along insulators surrounding conductors in liquid argon," JINST 11 no. 03, (2016) P03026, arXiv:1506.04185 [physics.ins-det]. http://stacks.iop.org/1748-0221/11/i=03/a=p03026.

[9] R. Brun and F. Rademakers, "ROOT - An Object Oriented Data Analysis Framework, Proc. AIHENP '96 Workshop," Nucl. Instrum. Meth. A 389 (1997) 81-86.

[10] W. Weibull, “A statistical distribution function of wide applicability," J. Appl. Mech 18 (1951) 293-277.

[11] J. Gerhold, M. Hubmann, and E. Telser, "Gap size effect on liquid helium breakdown," Cryogenics 34 no. 7, (1994) 579.

[12] M. Auger, A. Blatter, A. Ereditato, D. Goeldi, S. Janos, I. Kreslo, M. Luethi, C. Rudolf von Rohr, T. Strauss, and M. S. Weber, "On the Electric Breakdown in Liquid Argon at Centimeter Scale," JINST 11 no. 03, (2016) P03017, arXiv:1512.05968 [physics.ins-det].

[13] D. Swan and T. Lewis, "The influence of cathode and anode surfaces on the electric strength of liquid argon," Proc. Phys. Soc. 78 (1961) 448.

[14] G. Carugno et al., "Electron lifetime detector for liquid argon," Nucl. Instrum. Meth. A 292 no. 3, (1990) 580-584. http://www.sciencedirect.com/science/article/pii/0168900290901767. 